\title{
Docetaxel, Oxaliplatin and Capecitabine (TEX) triplet regimen as adjuvant chemotherapy in resected gastric adenocarcinoma
}

\author{
Divya Bala Thumaty ${ }^{1}$, Raju Titus Chacko ${ }^{1}$, Ajoy Oommen John ${ }^{1}$, Anjana Joel $^{1}$, Josh Thomas Georgy ${ }^{1}$, Myla Jacob² $^{2}$ Inian Samarasam² \\ Dipti Masih ${ }^{3}$, Rajesh Isaiah ${ }^{4}$, Visalakshi Jeyaseelan ${ }^{5}$ and Ashish Singh ${ }^{1}$
}

\author{
${ }^{1}$ Department of Medical Oncology, Christian Medical College, Ida Scudder Road, Vellore, TN, India \\ ${ }^{2}$ Department of Upper GI surgery, Division of Surgery, Christian Medical College, Vellore, India \\ ${ }^{3}$ Department of General Pathology, Christian Medical College, Vellore, India \\ ${ }^{4}$ Department of Radiotherapy, Christian Medical College, Vellore, India \\ ${ }^{5}$ Department of Biostatistics, Christian Medical College, Vellore, India
}

\section{Abstract}

Background: Adjuvant chemotherapy after surgery for gastric cancer improves survival but is difficult to administer due to poor tolerance. Combination chemotherapy with Docetaxel (Taxotere), Oxaliplatin (Eloxatin) and Capecitabine (Xeloda) (TEX) is used in the treatment of advanced gastric cancer. The efficacy and tolerability of this regimen (TEX) post resection of gastric cancer have not been studied.

Materials and methods: Patients diagnosed with gastric adenocarcinoma, post resection without any prior chemotherapy between July 2007 and May 2011 and treated with TEX regimen administered as T $35 \mathrm{mg} / \mathrm{m}^{2}$ and $E 50 \mathrm{mg} / \mathrm{m}^{2}$ on days (d) 1,8 and X $625 \mathrm{mg} / \mathrm{m}^{2}$ bid (twice daily) on d 1-14 every 21 days were included in this retrospective analysis. Patient's electronic medical records were studied and data on tolerance, progression-free survival (PFS) and overall survival (OS) was collected.

Results: Fifty-eight patients were treated with adjuvant TEX chemotherapy, majority 40 (68\%) had distal gastric cancer. All patients underwent a D1 gastrectomy, and resection was performed for 44 (75\%). Only 14 (24\%) patients had more than 15 nodes studied in the resected specimen. Distribution for stages I, II and III is 14 (24\%), 30 (52\%) and 14 (24\%), respectively. After a median follow-up of 40 months, the 3-year relapse free survival was 58\% (95\% Cl: 42-68), and estimated median OS was 71 months $(95 \% \mathrm{Cl}$ : 19-123 months). Twenty-three (40\%) required dose reduction due to toxicity. Grade 3 or 4 toxicity was recorded for 22 (37\%). Half (52\%) of patients completed all planned chemotherapy of six cycles.

Conclusion: Post resection of gastric adenocarcinoma adjuvant triplet TEX chemotherapy is a feasible and effective outpatient regimen. Diarrhoea, neutropenia and neuropathy were the common dose limiting toxicity. Post-surgery only half the numbers of patients are able to complete all planned cycles.

Keywords: gastric cancer, stomach cancer, FLOT, TEX, adjuvant chemotherapy
Correspondence to: Ashish Singh Email: todrashish@gmail.com

ecancer 2021, 15:1292

https://doi.org/10.3332/ecancer.2021.1292

Published: $21 / 09 / 2021$

Received: 13/04/2021

Publication costs for this article were supported by ecancer (UK Charity number 1176307).

Copyright: (c) the authors; licensee ecancermedicalscience. This is an Open Access article distributed under the terms of the Creative Commons Attribution License (http:// creativecommons.org/licenses/by/4.0), which permits unrestricted use, distribution, and reproduction in any medium, provided the original work is properly cited. 


\section{Introduction}

Gastric cancer tops the list of cancers associated with deaths and disability-adjusted life-years amongst all the cancers in the recent Global Burden of Disease Study of the burden and incidence of cancers in India [1].

Surgery followed by adjuvant chemotherapy with fluoropyrimidine with or without platinum is considered as a standard treatment for operable gastric cancers [2]. Docetaxel in combination with Oxaliplatin and 5 Fluorouracil has improved survival and has become the standard perioperative chemotherapy regimen in fit patients [3]. Perioperative approach can make systemic chemotherapy available to all patients. Preoperative chemotherapy however may not be possible in the presence of a bleeding ulcer or gastric outlet obstruction. Tolerance reported to adjuvant chemotherapy following gastrectomy is low with only $45 \%-60 \%$ of patient being able to start and complete all planned cycles following surgery $[4,5]$.

For advanced gastric cancers, one of the ways to make triplet chemotherapy more tolerable without compromising on efficacy is to replace 5Flurouracil (5FU) with Capecitabine and administer a lower dose of Oxaliplatin and Docetaxel on D1 and day 8 [6-9]. The efficacy of this combination when administered post operatively is not known. In the present study, we report the outcomes with use of Docetaxel, Oxaliplatin, Capecitabine (TEX) regimen in adjuvant setting at a single centre, in south India.

\section{Methods}

This is a single centre, Institutional Review Board (IRB)-approved retrospective analysis of patients with gastric cancer who received TEX chemotherapy after surgery at our centre. All patients treated between May 2007 and April 2011 are included in this analysis. Patients satisfying all the following criteria were included in analysis: histologically proven gastric cancer who have undergone a complete resection (RO or R1) and received at least one cycle of chemotherapy in our department, with no evidence of metastatic disease on CT scan of abdomen and chest imaging.

The regimen consisted of Docetaxel administered at $35 \mathrm{mg} / \mathrm{m}^{2}$ on day 1 and day 8 , Oxaliplatin at $50 \mathrm{mg} / \mathrm{m}^{2}$ on days 1 and 8 and Capecitabine at $1,250 \mathrm{mg} / \mathrm{m}^{2} /$ day, days $1-14$, cycle repeated every 21 days. Toxicity assessment was done at every patient visit and recorded as per $\mathrm{NCl}-$ CTCAE version 4.0. End points assessed were relapse free survival defined as time from diagnosis to relapse or death and overall survival (OS) defined as time from diagnosis to death.

\section{Clinical data collection and statistics}

For this study, demographic data and baseline clinical data were collected retrospectively from the electronic medical records and patients were contacted by telephone regarding the disease status and survival. Follow-up data was updated till September 2019. All data were entered in SPSS Statistics version 21 (IBM) and used for analysis. Descriptive statistics including median, frequency and percentage for categorical variables was used to describe age, gender distribution, treatment and response to treatment. Median event-free survival (EFS) and OS were calculated using Kaplan-Meier estimates.

\section{Results}

Of all the inpatient records reviewed between May 2007 and April 2011, 384 patients were admitted for administration of chemotherapy for gastric adenocarcinoma (including adjuvant, neoadjuvant and metastatic disease). Adjuvant chemotherapy was administered to 93 patients, of which 18 patients received only a doublet chemotherapy with Oxaliplatin and 5-Flurouracil while 75 patients received TEX. Fifty-eight patients who fulfilled the inclusion criterion with available date were included in this retrospective study. Patient and disease characteristics are presented in Table 1. Median age at presentation was 53 years (range: 30-70 years). Gastric cancer as expected was commoner in men (69\%), and majority (77\%) had distal tumours. Patients with proximal tumour were more likely to receive neoadjuvant chemotherapy and therefore represent only $22 \%$ in this study. 
Surgical findings and final pathological stage are presented in Table 2. Staging was I, II and III in 14 (24\%), 30 (52\%) and 14 (24\%), respectively; There were four patients with stage 1a tumour who received chemotherapy - indications being - distal margin positivity and lymphovascular invasion. Forty-four (76\%) patients had $<15$ nodes harvested from the surgical specimen.

The median time to start of adjuvant chemotherapy was 3 weeks. After a median follow-up of 40 months, the 3-year relapse free survival was 58\% (95\% Cl: 42-68), and estimated median OS was 71 months (95\% Cl: 19-123 months) as presented in Figure 1.

Table 1. Baseline characteristics.

\begin{tabular}{|l|l|}
\hline \multicolumn{1}{|c|}{ Patient and tumour characteristic } & \multicolumn{1}{|c|}{ Total $\boldsymbol{n}=\mathbf{5 8}(\%)$} \\
\hline Age (Median, range) & 53 years, Range: $30-70$ years \\
\hline Gender & \\
Males & $40(68 \%)$ \\
Females & $18(31 \%)$ \\
\hline Site & $45(78 \%)$ \\
Distal & $13(22 \%)$ \\
Proximal & \\
\hline Histology & $29(50 \%)$ \\
Poorly differentiated & $26(45 \%)$ \\
Moderately differentiated & $3(5 \%)$ \\
\hline Well differentiated & $12 / 58(20 \%)$ \\
\hline Signet ring histology & $31 / 58(53 \%)$ \\
\hline LVI present & $19 / 58(32 \%)$ \\
\hline PNI present & \\
\hline
\end{tabular}

LVI: lymphovascular invasion; PNI: perineural invasion

Table 2. Surgical details

\begin{tabular}{|l|l|}
\hline \multicolumn{1}{|c|}{ Surgical details } & \multicolumn{1}{|c|}{ Number (\%) } \\
\hline Type of gastrectomy & $N=58$ \\
Total & $13(22 \%)$ \\
Subtotal & $38(65 \%)$ \\
Distal & $7(12 \%)$ \\
\hline Lymph node dissection & $n=5411(20 \%)$ \\
D1 & $42(77 \%)$ \\
D1 + & 0 \\
D2 & 1 \\
D0 & \\
\hline No of lymph node dissected & $n=57$ \\
$<15$ & 43 \\
$\geq 15$ & 14 \\
\hline Completeness of resection & $N=58$ \\
R0 & 44 \\
R1 & 12 \\
R2 & 0 \\
Not known & 2 \\
\hline Final path TNM stage & $N=58$ \\
1 & 14 \\
2 & 30 \\
3 & 14 \\
4 & 0 \\
\hline
\end{tabular}




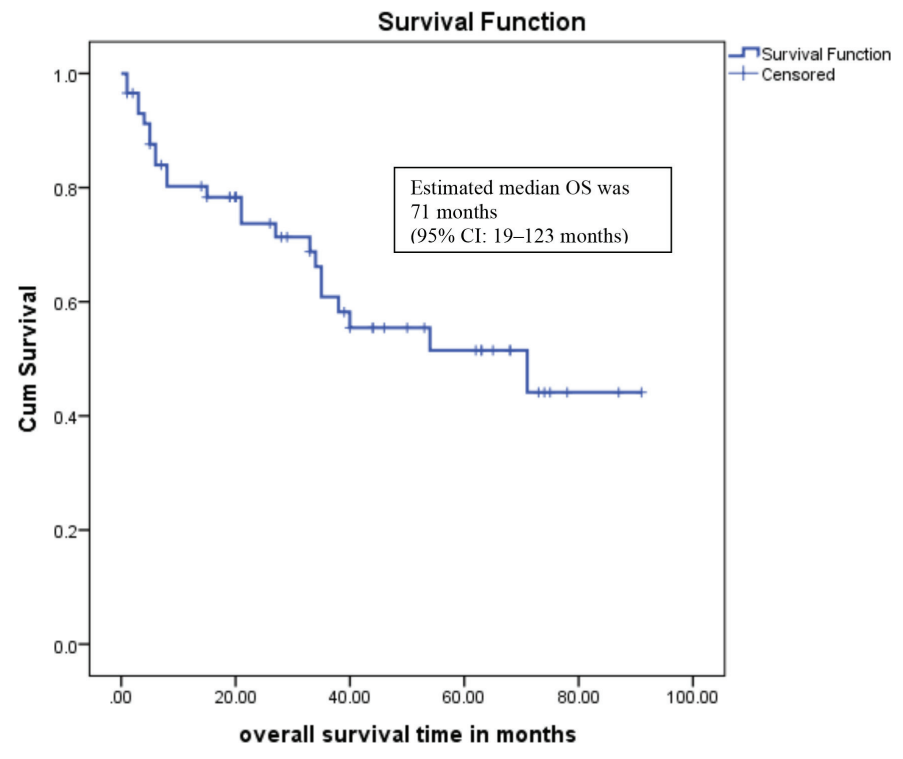

Figure 1. Overall survival.

Table 3. Adverse events

\begin{tabular}{|l|l|l|}
\hline \multicolumn{1}{|c|}{ Toxicity } & \multicolumn{1}{|c|}{ Grade 1-2, $\mathbf{n}(\%)$} & \multicolumn{1}{c|}{ Grade 3-4, $\mathbf{n = 2 2 ( 3 7 \% )}$} \\
\hline Anaemia & $20(34 \%)$ & $6(10 \%)$ \\
\hline Thrombocytopenia & $14(24 \%)$ & $4(6 \%)$ \\
\hline Febrile neutropenia & $2(3 \%)$ & $4(6 \%)$ \\
\hline Diarrhoea & $16(28 \%)$ & $8(13 \%)$ \\
\hline Neuropathy & $12(20 \%)$ & $5(9 \%)$ \\
\hline Hand foot syndrome & $24(41 \%)$ & $6(10 \%)$ \\
\hline Hypersensitivity & $3(5 \%)$ & $1(2 \%)$ \\
\hline
\end{tabular}

Toxicity recorded is presented in Table 3.

Twenty-three (40\%) required dose reduction due to toxicity. Grade 3 or 4 toxicity was recorded for 22 (37\%). 52\% of patients completed all planned chemotherapy. Ten (17\%) patients discontinued chemotherapy due to grade 3 toxicity.

\section{Discussion}

About two decades ago, the intergroup 0116 trial reported improved survival with adjuvant chemoirradiation post resection for gastric adenocarcinoma [10]. Locoregional relapse reduction accounted for majority of overall relapse reduction [11]. Since then, trials have confirmed that after a radical resection for gastric cancer and adjuvant chemotherapy, addition of chemo irradiation does not improve survival [5, 12, 13]. Oral chemotherapy with S1 or Capecitabine as a single agent or combination with Oxaliplatin is effective and is the standard treatment post resection $[14,15]$. In another study for early stage gastric cancers, Epirubicin when combined with Cisplatin and Capecitabine did not improve outcomes over the doublet alone [16]. We designed the TEX adjuvant regimen based on the findings from published reports that showed addition of Docetaxel to platinum and 5FU resulted in better response rates and PFS in multiple reports [7,8]. 
The assumption was Docetaxel may be better than Epirubicin in early gastric cancer. Later on it was shown that Docetaxel in combination with Oxaliplatin and infusional 5 Fluorouracil (FLOT) was superior to Epirubicin, Cisplatin, Flurouracil (ECF) in patients receiving perioperative chemotherapy for non-metastatic gastric cancer [3]. A triplet regimen like FLOT or TEX is expected to improve outcomes, provided it can be administered safely to patients post major surgery like gastrectomy. Administration of chemotherapy post-surgery is poorly tolerated with only $45 \%-60 \%$ of patients able to start chemotherapy after surgery and less number are able to complete the entire planned therapy [4, 5] Replacing 5-FU with Capecitabine obviates the need for an infusion pump and central line, associated with increase in cost and catheter related complications, both drugs have a similar incidence of toxicities, though a different profile. TEX is easy to administer as an outpatient treatment as compared to FLOT. Postoperatively more than six cycles are expected to be poorly tolerated due to neuropathy, hand foot syndrome and diarrhoea.

Seventy-eight percent of patients had a distal cancer; upfront surgery is more common for distal cancers for reasons such as obstruction or bleeding. Perioperative chemotherapy is preferred for proximal lesions in the absence of contraindications for chemotherapy. Surgery performed was not D2 resection for any of our patients. However, D1 or an extended D1 gastrectomy is associated with lesser morbidity without any significant compromise on survival [17]. 12 (21\%) patients had R1 resection. Adjuvant chemo irradiation is poorly tolerated and is not standard of care at our centre. Our results indicate that TEX is feasible and is an effective regimen with $58 \%$ of patients being free of disease recurrence at 3 years after diagnosis. These results are despite the fact that $48 \%$ of patients were unable to complete all assigned therapy. The median OS was 71 months, higher as compared to the published literature. This is probably due to the inclusion of only fit patients and early stage disease (stage 1, 2 amounting to $77 \%$ of patients) who are able to start chemotherapy and a smaller sample size.

Most relapses were distant, and data on local only relapse rate is not available.

Fatigue, diarrhoea, hand foot syndrome, neutropenia and neuropathy were the major toxicity with this regimen. There was one treatment related death due to febrile neutropenia and septic shock that occurred soon after the first dose. Dihydropyrimidine dehydrogenase (DPD) enzyme deficiency testing may predict such severe toxicity with fluoropyrimidines but isn't yet done for every patient at our centre. Cumulative toxicities like hand foot syndrome, fatigue and neuropathy developed over time and were the main reason for dose reduction and early discontinuation of treatment. From this experience, we suggest that a modification in the schedule which allows infusion of Oxaliplatin and Docetaxel every biweekly in order to reduce toxicity and make the regimen tolerable to more patients who are at risk of relapse.

The limitations of our study include the retrospective nature, absence of a control arm and inclusion of only patients who were able to start chemotherapy after surgery.

\section{Conclusion}

Post resection of gastric adenocarcinoma adjuvant TEX is a feasible outpatient triplet chemotherapy regimen with acceptable results. Diarrhoea, neutropenia and neuropathy are the common toxicity requiring dose adjustments. Shortening the duration of adjuvant treatment by use as perioperative treatment may reduce toxicity, improve compliance and should be studied.

\section{Acknowledgments}

Nil.

\section{Conflicts of interest}

Nil.

\section{Funding}

Source(s) of support: CMC Fluid Research Grant. 


\section{References}

1. Dhillon PK, Mathur P, and Nandakumar A, et al (2018) The burden of cancers and their variations across the states of India: the Global Burden of Disease Study 1990-2016 Lancet Oncol 19(10) 1289-1306 https://doi.org/10.1016/S1470-2045(18)30447-9

2. Paoletti X, Oba K, and Burzykowski T, et al (2010) Benefit of adjuvant chemotherapy for resectable gastric cancer: a meta-analysis J Am Med Assoc 303(17) 1729-1737 https://doi.org/10.1001/jama.2010.534

3. Al-Batran SE, Homann N, and Pauligk C, et al (2019) Perioperative chemotherapy with fluorouracil plus leucovorin, oxaliplatin, and docetaxel versus fluorouracil or capecitabine plus cisplatin and epirubicin for locally advanced, resectable gastric or gastro-oesophageal junction adenocarcinoma (FLOT4): a randomised, phase 2/3 trial Lancet 393(10184) 1948-1957 https://doi.org/10.1016/S01406736(18)32557-1 PMID: 30982686

4. Mirza A, Pritchard S, and Welch I (2013) The postoperative component of MAGIC chemotherapy is associated with improved prognosis following surgical resection in gastric and gastrooesophageal junction adenocarcinomas Int J Surg Oncol 2013 PMID: 24163764 PMCID: 3791565

5. Cats A, Jansen EP, and van Grieken NC, et al (2018) Chemotherapy versus chemoradiotherapy after surgery and preoperative chemotherapy for resectable gastric cancer (CRITICS): an international, open-label, randomised phase 3 trial Lancet Oncol 19(5) 616-628 https://doi.org/10.1016/S1470-2045(18)30132-3 PMID: 29650363

6. Stein A, Arnold D, and Thuss-Patience PC, et al (2014) Docetaxel, oxaliplatin and capecitabine (TEX regimen) in patients with metastatic gastric or gastro-esophageal cancer: results of a multicenter phase I/II study Acta Oncol 53(3) 392-398 https://doi.org/10.3109/0284 186X.2013.833346

7. Evans D, Miner T, and Akerman P, et al (2007) A phase I study of docetaxel, oxaliplatin, and capecitabine in patients with metastatic gastroesophageal cancer Am J Clin Oncol 30(4) 346-349 https://doi.org/10.1097/COC.0b013e318042d582 PMID: 17762433

8. Grothe W, Hofheinz RD, and Mantovani Loeffler L, et al (2006) Phase I trial of docetaxel, oxaliplatin and capecitabine (TEX) in patients with metastatic gastric cancer J Clin Oncol 24(18_suppl) 14051-14051 https://doi.org/10.1200/jco.2006.24.18_suppl.14051

9. Ajani JA, Moiseyenko VM, and Tjulandin S, et al (2007) Clinical benefit with docetaxel plus fluorouracil and cisplatin compared with cisplatin and fluorouracil in a phase III trial of advanced gastric or gastroesophageal cancer adenocarcinoma: the V-325 study group J Clin Oncol 25(22) 3205-3209 https://doi.org/10.1200/JCO.2006.10.4968 PMID: 17664467

10. Macdonald JS, Smalley SR, and Benedetti J, et al (2001) Chemoradiotherapy after surgery compared with surgery alone for adenocarcinoma of the stomach or gastroesophageal junction N Engl J Med 345(10) 725-730 https://doi.org/10.1056/NEJMoa010187 PMID: 11547741

11. Smalley SR, Benedetti JK, and Haller DG, et al (2012) Updated analysis of SWOG-directed intergroup study 0116: a phase III trial of adjuvant radiochemotherapy versus observation after curative gastric cancer resection J Clin Oncol 30(19) 2327-2333 https://doi. org/10.1200/JCO.2011.36.7136 PMID: 22585691 PMCID: 4517071

12. Park SH, Zang DY, and Han B, et al (2019) ARTIST 2: interim results of a phase III trial involving adjuvant chemotherapy and/or chemoradiotherapy after D2-gastrectomy in stage II/III gastric cancer (GC) J Clin Oncol 37(15_suppl) 4001-4001 https://doi.org/10.1200/ JCO.2019.37.15_suppl.4001

13. Lee J, Lim DH, and Kim S, et al (2011) Phase III trial comparing capecitabine plus cisplatin versus capecitabine plus cisplatin with concurrent capecitabine radiotherapy in completely resected gastric cancer with D2 lymph node dissection: the ARTIST trial J Clin Oncol 30 268-273 https://doi.org/10.1200/JCO.2011.39.1953 PMID: 22184384

14. Bang YJ, Kim YW, and Yang HK, et al (2012) Adjuvant capecitabine and oxaliplatin for gastric cancer after D2 gastrectomy (CLASSIC): a phase 3 open-label, randomised controlled trial Lancet 379(9813) 315-321 https://doi.org/10.1016/S0140-6736(11)61873-4 PMID: 22226517 
15. Sakuramoto S, Sasako M, and Yamaguchi T, et al (2007) Adjuvant chemotherapy for gastric cancer with S-1, an oral fluoropyrimidine $N$ Engl J Med 357(18) 1810-1820 https://doi.org/10.1056/NEJMoa072252 PMID: 17978289

16. Alderson D, Cunningham D, and Nankivell M, et al (2017) Neoadjuvant cisplatin and fluorouracil versus epirubicin, cisplatin, and capecitabine followed by resection in patients with oesophageal adenocarcinoma (UK MRC OE05): an open-label, randomised phase 3 trial Lancet Oncol 18(9) 1249-1260 https://doi.org/10.1016/S1470-2045(17)30447-3 PMID: 28784312 PMCID: 5585417

17. Memon MA, Subramanya MS, and Khan S, et al (2011) Meta-analysis of D1 versus D2 gastrectomy for gastric adenocarcinoma Ann Surg 253(5) 900-911 https://doi.org/10.1097/SLA.0b013e318212bff6 PMID: 21394009 